Villaibn H. y cols.

Rev. Chll. Podfatr. 64 (2); 124-128, 1993

\title{
Efecto a corto plazo del contacto precoz piel a piel sobre la lactancia materna en recién nacidos de término sanos
}

\author{
Hemán Villalón U.’; Patricia Alvarez C. ${ }^{1}$ \\ Short term effects of early skin-to-skin contact (kangaroo care) \\ on breast feeding in healthy full term newborns
}

\begin{abstract}
To evaluate the effects on brest feeding of early sklnto-skin conlact 119 pairs of mothers and thelr respective newborn infants were observed thraugh a randomized prospective and controlled triol in which 59 pairs underwent eorly skintor skin conlact. Breast leeding and mother's subjective perception of selfconfidence were evoluated. This trial was carried out at the Regional Hospital of Coytraique, Chile (western Patagonia), from december 1901 throughout march 1992. Significant differences were found in the paoportion of breast led infants 24 hours after birth $189.9 \%$ in the SSC group vs $03.3 \%$ in controls, $p<0.0011$, a hospilal discharge $193.3 \%$ vs $60.7 \%, p<0.001$ ) and at oge 14 doys $178.8 \%$ vs $50.2 \%$, $p<0.021$. These differences were not dependent of pority. Significont differences were olso noled in molernol selfconfidence about theil child care al discharge $[89.8 \%$ vs $53.3 \%, p<0.001\}$ and 14 days post partum $\{97 \%$ vs $71.8 \%, p<0.011$, this being more remorkable among primiporous mothers. These differences were not influenced by educational level. Undoubledly, selfcofidence is a very subjective matter to be evaluoled with such a simple method, therefore, futher studies are needed. Nevertheless, this is a vefy interesting way to encourage breast feeding.
\end{abstract}

(Key wordst newborn, early skin-to-skin conlact, breast feeding, maternal selfconfidence)

La XI Región de Aysén tiene características diferentes al resto del país en cuanto a su baja densidad poblacional $\left(0,5\right.$ habitante $\left./ \mathrm{km}^{2}\right)$ y alta nuralidad $(60 \%)$, con una población dispersa y de difícil acceso. Por esta razón, los esfuerzos han estado orientados principalmente a aumentar la atención profesional del parto, lográndose excelente cobertura $(99,23 \%)$. Sin embargo, todavía son altas las cifras de recién nacidos (RN) con bajo peso de nacimiento $(6,5 \%)$ y peso insuficiente $(21,8 \%)^{1}$. Estos resultados motivan nuestro interés en el fomento de la laciancia materna, lo cual se ve dificultado por la dispersión de la población, impidiendo adecuados planes educativos prenatales.

Se diseffó una estrategia para estimular la lactancia materna, basada en experiencias publi-

1. Unidad de Cuidados Intensivos Pediátricos y Neonatales, Hospital Regional de Coyhaique, XI Regiớn. cadas $^{2}{ }^{3}$, fomentando el contacto precoz prolongado piel a piel (CPPP) entre el RN y su madre. Se aprovechó este trabajo para evaluar la sensación de seguridad e inseguridad de las madres en relación a sus hijos. La experiencia se analizó a través de un estudio prospectivo controlado.

Un estudio previo, realizado en nuestro hospital, demostró la seguridad del método en RN de término sanos

\section{Pacientes y Métodos}

Se estudiaron 119 RN y sus madres, en el Servicio de Matemidad del Hospital Regional de Coyhaique, en el perodo diciembre 1991-marzo 1992. Los RN incluidos tenían edad gestacional pediátrica de 38 a 41 semanas; peso de nacimiento entre 2500 y $4250 \mathrm{~g}$; Apgar al primer y quinto minuto igual o superior a 4 y nacidos por parto eutócico vaginal. Se excluyeron los $\mathrm{RN}$ con antecedentes de rotura de membranas superior a 12 horas, sospecha de infección bacteriana perinatal y hemograma sugerente (según criterios de Radwell) o cultivo positivo, malformaciones, síndrome de dificultad respiratoria, cartiopatjas o hipoglicemia. 
l.a mucstra se distribuyó en forma alealoria oblenićndose así 59 RN para el grupo de CPPP y 60 para el grupo control. Se analizaron los datos matemos (edad, paridad, ruralidad y escolaridad), del parto (ticmpo de rotura de membranas, anestesia y desaceleraciones de latidos cardiofetales) y del RN (scro, peso y edad gestacional estimada según Parkin). Para evaluar la laclancia matema se observó presencia o ausencia de succión al nacumiento, 2 y 4 horas posteriores al parto. Se registró la modalidad de alimentación a las $24 \mathrm{~h}$ de vida, al momento del alta y a los 14 días, cslifiéndola como adecuada en caso de alimentación con pecho en foma exclusiva, c inadecuada en caso de requerir alimentación con fórmula en forma total o parcial. A todas las madres se les preguntó, al momento del alı y en el control de los 14 dias de edad de los hijos: "¿se siente Ud. segura con su hijo?", lo gue siempre fue realizado por la misma persona sin saber a qué grupo correspondía la paciente y sin cambiar la fótmula de la pregunta. Las respuestas se clasificaron śblo cn seguridad o inseguridad.

Los reciér nacidos del gnpo control, después del nacimicnto, fueron secados y sometidos a mediciones antropomćtricas, luego vestidos y dejados en la sala de atcnción inmediata (SAl) durante cuatro horas, en que se controlaron frccuencia cardiaca, respiratoria y temperatura, Los niños del grupo de contacto precoz prolongado piel a piel, des- pućs de secados y medidos fueron vestidos sólo con un pañal, entregados a sus madres, puestos cntre las mamas y cubiertos sólo por una sábana, iniciándose un contacto piel a piel y la precoz succión del pezón. Este contacto se prolongó por cuatro horas más en las salas de la matemidad donde se realizaron los registros de parámerros fisiologicos mencionados en hos niños control.

El análisis estadístico de los rcsultados se realizó mediante las pruebas de chi cuadrado para comparación de proporciones y porcentajes, análisis de varianza para la determinación de la prueba de Student para promedios. Se establecjó como limite de confianza niveles inferiores a $5 \%$.

\section{Resultados}

Los grupos control y estudio eran similares en cuanto a edad matema, tipo de anestesia, sexo del RN, paridad, ruralidad, escolaridad, edad gestacional, peso de nacimiento, tiempo de rotura de membranas y desaceleraciones de latidos cardiofetales (tabla 1).

\section{Tabla 1}

Características estudiadas en 119 pacientes distribuidos en grupos CPPP y control. Hospital Regional de Coyhaique (dicienbre 1991-marzo 1992)

\begin{tabular}{|c|c|c|c|c|c|}
\hline \multirow[b]{2}{*}{$\begin{array}{l}\text { Edad materna (años) } \\
\text { T. rotura membrana (h) }\end{array}$} & \multicolumn{2}{|c|}{$\begin{array}{c}\text { CPPP } \\
\text { (n: 59) }\end{array}$} & \multicolumn{2}{|c|}{$\begin{array}{l}\text { Control } \\
\text { (n: 60) }\end{array}$} & \multirow{2}{*}{$\begin{array}{l}\mathbf{p} \\
\text { ns } \\
\text { ns }\end{array}$} \\
\hline & $\underset{2,86}{25,9} \pm$ & $\begin{array}{l}5.9 \\
3.34\end{array}$ & $\begin{array}{l}25,3 \\
3,80\end{array}$ & $\begin{array}{l} \pm 5,9 \\
\pm 7,34\end{array}$ & \\
\hline \multicolumn{6}{|l|}{ Anestesia } \\
\hline $\begin{array}{l}\text { Peridural } \\
\text { Local } \\
\text { Sin anestesia }\end{array}$ & $\begin{array}{l}15,2 \% \\
61,0 \% \\
23,8 \%\end{array}$ & $\begin{array}{l}(9 / 59) \\
(36,59) \\
(14 / 59)\end{array}$ & $\begin{array}{r}8.3 \% \\
63,3 \% \\
28,3 \%\end{array}$ & $\begin{array}{l}(5 / 60) \\
(38 / 60) \\
(17 / 60)\end{array}$ & $\begin{array}{l}\text { ns } \\
\text { ns } \\
\text { ns }\end{array}$ \\
\hline \multicolumn{6}{|l|}{ Sexo } \\
\hline $\begin{array}{l}\text { Masculino } \\
\text { Femenino }\end{array}$ & $\begin{array}{l}49,1 \% \\
50,9 \%\end{array}$ & $\begin{array}{l}(29 / 59) \\
(30 / 59)\end{array}$ & $\begin{array}{l}46,7 \% \\
53,3 \%\end{array}$ & $\begin{array}{l}(28 / 60) \\
(32 / 60)\end{array}$ & $\begin{array}{l}\text { ns } \\
\text { ns }\end{array}$ \\
\hline \multicolumn{6}{|l|}{ Paridad } \\
\hline $\begin{array}{l}\text { Primigestas } \\
\text { Muitíparas }\end{array}$ & $\begin{array}{l}27,1 \% \\
72,9 \%\end{array}$ & $\begin{array}{l}(16 / 59) \\
(43 / 59)\end{array}$ & $\begin{array}{l}28,3 \% \\
71,7 \%\end{array}$ & $\begin{array}{l}(17 / 60) \\
(43 / 60)\end{array}$ & $\begin{array}{l}\mathrm{ns} \\
\mathrm{ns}\end{array}$ \\
\hline Ruralidad & $30,5 \%$ & $(18 / 59)$ & $30,0 \%$ & $(18 / 60)$ & ns \\
\hline \multicolumn{6}{|l|}{ Escolaridad } \\
\hline $\begin{array}{l}\leq 8^{\circ} \text { básico } \\
\geq 1^{\underline{9} \text { educación media }}\end{array}$ & $\begin{array}{l}40,7 \% \\
59,3 \%\end{array}$ & $\begin{array}{l}(24 / 59) \\
(35 / 59)\end{array}$ & $\begin{array}{l}51,7 \% \\
48,3 \%\end{array}$ & $\begin{array}{l}(31 / 60) \\
(29 / 60)\end{array}$ & $\begin{array}{l}\text { ns } \\
\text { ns }\end{array}$ \\
\hline $\begin{array}{l}\text { Edad gestacional (sem) } \\
\text { Peso }(\mathrm{g}) \\
\text { Desaceleraciones LCF }\end{array}$ & $\begin{array}{c}39,1 \pm \\
3442 \\
6,8 \%\end{array}$ & $\begin{array}{l}0,8 \\
296 \\
(4 / 59)\end{array}$ & $\begin{array}{c}38,3 \\
3346 \\
11.7 \%\end{array}$ & $\begin{array}{l} \pm 1,0 \\
\pm 363 \\
(7 / 60)\end{array}$ & $\begin{array}{l}n s \\
\text { ns } \\
\text { ns }\end{array}$ \\
\hline
\end{tabular}


El análisis de la lactancia matema no demostró diferencias estadísticamente significativas en la incidencia de succión, reflejo de succión al nacimiento, a las 2 y 4 horas posteriores al nacimiento, calificándose como adecuada en $100 \%$ (n: 59) del grupo estudio y en 98,3\% (n: 60) del grupo control. Se encontraron diferencias estadísticamente significativas en la incidencia de lactancia materna exclusiva a las 24 horas de vida $(89,9 \%$ en el grupo estudio y $63,3 \%$ en el grupo control) y $93,3 \%$ en el momento del alta para el grupo CPPP y $66.7 \%$ en el grupo control. Sólo se logró controlar a los 14 días al $56 \%$ (n: 33 ) del grupo estudio y al $53,3 \%$ (n: 32) del grupo control, manteniéndose diferencias significativas. Las diferencias no se modifican al analizarlas según paridad, excepto en el grupo de las primigestas en el control de los 14 días, en que el resultado obtenido puede corresponder a una distorsión debido a la baja casuística evaluada (tabla 2).

En la sensación de seguridad declarada por las madres se registraron importantes diferencias entre ambos grupos, incluso en el control de los 14 días, las que se mantuvieron independientes de la paridad y escolaridad (tabla 3 ).

\section{Comentario}

Los resultados obtenidos en este estudio son concordantes con los comunicados por otros au- tores $^{2,3}$ y apoyan las recomendaciones acerca de la alimentación en el primer año de vida ${ }^{5}$.

Si bien es cierto que no habia diferencias en la capacidad de succión de los RN aí nacer ni cn las horas siguientes, éstas eran significativas en los porcentajes de niños con lactancia materna $\mathrm{cx}$ clusiva a las 24 horas de vida y al alta. A pesar de que sólo $56 \%$ de los niños del grupo esludio y $53,3 \%$ del grupo control pudieron ser evaluados a los 14 días, se mantenían aún diferencias estadísticas cntre ambos grupos, cxcepto entre primigestas, probablemente por el bajo número đe niños controlados. Un seguimiento más prolongado scrá necesario para evaluar los efectos a largo plazo sobre este parámetro. Sin embargo, este grupo es el más beneficiado con el método al momento del alta, por las diferencias observadas en la ocurrencia de lactancia materna exclasiva.

Muy llamativo es lo observado con respecto a la sensación de seguridad o inseguridad de las madres frente a sus hijos. Aunque el procedimicnto utilizado -por no emplear una escala tendiente a objetivar dicho parámetro- no es adecuado, el resultado no debc ser desestimado, sino por el contrario, molivar futuros estudios tendiente a profundizar en el conocimiento de la influencia psicologica del contacto piel a piel entre la madre y su RN. El análisis según escolaridad sugiere una infuencia cultural negativa sobre este aspecto. Si bien es cierto que se observan diferen-

Tabla 2

Análisis comparativo de alimentación con pecho matemo exclusivo y según paridad en grupos CPPP y control. Hospital Regional de Coyhaique (diciembre 1991-marzo 1992)

\begin{tabular}{lccc}
\hline & CPPP & Control & P \\
\hline A las 24 horas & $89,9 \%(54 / 59)$ & $63,3 \%(38 / 60)$ & $\mathrm{p}<0,001$ \\
Al alta & $93.2 \%(55 / 59)$ & $66,7 \%(40 / 60)$ & $\mathrm{p}<0,001$ \\
A los 14 días & $78,8 \%(27 / 33)$ & $56,2 \%(18 / 32)$ & \\
Paridad & & & \\
Primigestas & & & \\
A las 24 horas & $81,2 \%(13 / 16)$ & $52,9 \%(9 / 17)$ & $\mathrm{P}<0,05$ \\
Al alta & $93,8 \%(15 / 16)$ & $52,9 \%(9 / 17)$ & $\mathrm{P}<0,01$ \\
A los 14 días & $77,7 \%(7 / 9)$ & $50,0 \%(6 / 10)$ & $\mathrm{ns}$ \\
Multiparas & & & \\
A las 24 horas & & $65.1 \%(29 / 43)$ & $\mathrm{P}<0,001$ \\
Al alta & $95.3 \%(41 / 43)$ & $72,1 \%(31 / 43)$ & $\mathrm{P}<0,02$ \\
A los 14 dias & $93,0 \%(40 / 43)$ & $54,5 \%(12 / 22)$ & $\mathrm{P}<0,02$ \\
\hline
\end{tabular}


Tabla 3

Análisis comparativo sobre la sensación de seguridad de las madres frente a su RN y desglosado según paridad y escolaridad de los grupos CPPP y control. Hospital Regional de Coyhaique (diciembre 1991-marzo 1992)

\begin{tabular}{|c|c|c|c|}
\hline & CPPP & Control & $p$ \\
\hline Al alta & $89,8 \%(53 / 59)$ & $53,3 \%(32 / 60)$ & $p<0,001$ \\
\hline A los 14 días & $97,0 \%(32 / 33)$ & $72,8 \%(23 / 32)$ & $p<0,01$ \\
\hline \multicolumn{4}{|l|}{ Paridad } \\
\hline $\begin{array}{l}\text { Al alta } \\
\text { A los } 14 \text { días } \\
\text { Multíparas }\end{array}$ & $\begin{array}{r}93,8 \% \\
100,0 \% \quad(15 / 16)\end{array}$ & $\begin{array}{ll}35,3 \% & (6 / 17) \\
60,0 \% & (6 / 10)\end{array}$ & $\begin{array}{l}P<0,001 \\
p<0,01\end{array}$ \\
\hline $\begin{array}{l}\text { A } 1 \text { alta } \\
\text { A los } 14 \text { días }\end{array}$ & $\begin{array}{l}88,4 \%(38 / 43) \\
95,8 \%(23 / 24)\end{array}$ & $\begin{array}{l}60,4 \%(26 / 43) \\
77,3 \%(17 / 22)\end{array}$ & $\begin{array}{l}\mathrm{P}<0,01 \\
\mathrm{P}<0,05\end{array}$ \\
\hline \multicolumn{4}{|l|}{ Escolaridad } \\
\hline $\begin{array}{l}\leq 8^{2} \text { básico } \\
\text { Al alta } \\
\text { A los } 14 \text { días } \\
\geq 1^{2} \text { educación media }\end{array}$ & $\begin{array}{r}95,8 \%(23 / 24) \\
100,0 \%(15 / 15)\end{array}$ & $\begin{array}{l}67.7 \%(31 / 31) \\
76.5 \%(13 / 17)\end{array}$ & $\begin{array}{l}p<0,01 \\
p<0,02\end{array}$ \\
\hline $\begin{array}{l}\text { Al alta } \\
\text { A los } 14 \text { dias }\end{array}$ & $\begin{array}{l}94,3 \%(33 / 35) \\
94,4 \%(17 / 18)\end{array}$ & $\begin{array}{l}34,5 \%(10 / 29) \\
53,3 \% \quad(8 / 15)\end{array}$ & $\begin{array}{l}P<0,001 \\
p<0,01\end{array}$ \\
\hline
\end{tabular}

cias significativas en todos los grupos, nuevainente las primigestas son quienes más se benefician en la actitud frente a su hijo después de experimentar el CPPP, lo cual coincide con otras publicaciones ${ }^{2}$, resultando muy interesante la experiencia en este grupo de pacientes, donde el impacto del método es más relevantc. Resultados similares ban sido publicados en la literatura extranjera, aunque no en RN de término6.

Este estudio motiva a buscar más conocimiento y aplicación del método como esırategia a corto plazo para fomentar el apego madre-hijo 7 y cstimular la lactancia materna a través de un método sencillo, natural y gratificante.

\section{Resumen}

Ciento diecinueve madres y sus recién nacidos fueron estudiados a través de un estudio prospectivo aleatorio controlado, cn el Hospital Regional de Coyhaique, en el período entre dicicmbre 1991 y marzo 1992, con el propósito de evaluar los efectos del contacto precoz piel a piel sobre la lactancia natural y la sensación de seguridad de las madres con respecto a su hijo. Cincuenta y nueve parejas de madre e hijo fueron expuestas a contacto precoz piel a picl y scsenta al procedimiento corriente de observación por separado en las primeras cuatro horas de vida, evaluándose la alimentación con pecho exclusivo y la sensación subjetiva de seguridad frente al recién nacido. Se encontraron diferencias significativas en la incidencia de alimentación natural a las 24 horas después del nacimiento $(89,9 \%$ en el grupo contacto vs $63,3 \%$ en el grupo control, p $<0,001)$, al alta $(93,2 \%$ vs $66,7 \%, \mathrm{p}<0,001)$ y a los 14 dias de vida $(78,8 \%$ vs $56,2 \%$ p $<0,001)$. Estas diferencias fueron indepcndientes de la paridad. También fueron significativas las observadas en la sensación subjetiva matcma de seguridad al alta $(89,8 \%$ vs $53,3 \% \mathrm{p}<0,001)$ y a los 14 dias después del parto $(97 \%$ vs $71,8 \% \mathrm{p}<$ $0.01)$, esto fue aún más importante en el grupo de las primíparas. La escolaridad no influyó en estas diferencias. Sin duda que la sensación de seguridad no puede ser evaluada adecuadamente con un método tan simple, por lo que se re- 
quieren estudios más profundos. Sin embargo, ésta es una interesante forma de estimular la laclancia materna.

(Palabras clave: Contacto piel a piel, lactancia natural.)

\section{Referencias}

1. Indicadores biodemograficos año 1991: Servicio Salud Aysén. XI Región, 1992.

2. Wiberg $B$, Humble $K$, De Chateas $P$ : Long-term effect on mother-infanl behaviour of cxtra contact during the first hour post parum. Follow up at three years. Scand J So: Med 1989; 17: 181-191.
3. Whitelew A: Crianza tipo canguro: iSolamente ura bonita experiencia o uл avance importante para los premalutos? Pedialrics (cd. esp.) 1990; 29:171-172.

4. Viitalón $H$, Alvarez P, Barría $E$ y cots.: Contacto P'recoz Piel a Piel: E[ccto sobre los parámetros fisiológicos en las cualro horas posteriores al parto, en recićn nacidos de témino sanos. Rev Chil Podiatr 1992; 63: 140144.

5. Radrigán ME, Juez $G$, Coronel F: Alimentación en el primer año de vida. Rev Chil Pediatr 1989; 60 (supl. 1): 9-19.

6. Affonso D. Woklberg V. Perssons B: Mother's reaction to kangaroo care. Infants Behav Dev 1988; $11: 4-6$.

7. Widstrom AM, Wahlberg V. Mathiesen AS et at.: Shortterm efects on carly suckling and touch of the nipple on matemal bchaviour. Early Hum Dcv 1990; 21: 153 163. 